\title{
The mitochondrial genome of the pinewood nematode (Bursaphelenchus xylophilus) lineage introduced in Europe
}

\author{
Cláudia Moreira ${ }^{1}$, Barbara van Asch ${ }^{1}$, Luís Fonseca ${ }^{2}$, Isabel Pereira-Castro ${ }^{1}$, Raquel Silva ${ }^{1}$, Luísa Azevedo ${ }^{1}$, \\ Manuel Mota ${ }^{3}$, Isabel Abrantes ${ }^{2}$, António Amorim ${ }^{1,4}$, and Filipe Pereira ${ }^{1}$

\begin{abstract}
${ }^{1}$ Institute of Molecular Pathology and Immunology, University of Porto (IPATIMUP), Porto, Portugal, ${ }^{2}$ Department of Life Sciences, IMAR-CMA, University of Coimbra, Coimbra, Portugal, ${ }^{3}$ NemaLab/ICAAM - Instituto de Ciências Agrarias e Ambientais Mediterrânicas, Universidade de Évora - Núcleo da Mitra, Évora, Portugal, and ${ }^{4}$ Faculty of Sciences, University of Porto, Porto, Portugal
\end{abstract}

\begin{abstract}
The pinewood nematode (PWN) Bursaphelenchus xylophilus is the causative agent of pine wilt disease and the greatest biological threat to conifer forests worldwide. Here we describe the near-complete mitochondrial DNA (mtDNA) sequence (12,945 bp) of the PWN lineage recently introduced in Europe. The absence of polymorphisms across the mtDNA of three Portuguese isolates suggests that a single mitochondrial lineage was introduced in southwestern Europe. We also found that Portuguese isolates have an incomplete stop codon (TA) at COX3, while the reference mtDNA from a South Korean isolate has a complete stop codon (TAA). Moreover, two insertion/deletion polymorphisms change the ND4 protein in a stretch of seven amino acids, and a polymorphic mononucleotide repeat alters the predicted structure of the tyrosine tRNA in different geographical isolates. Overall, the new PWN mtDNA sequence provides a basis for studying the European dispersion of this important invasive species.
\end{abstract}

\section{Keywords}

Bursaphelenchus xylophilus, incomplete stop codon, indel, mitochondrial DNA, mononucleotide repeat, pine wilt disease

\author{
History \\ Received 10 May 2013 \\ Revised 21 May 2013 \\ Accepted 24 May 2013 \\ Published online 10 July 2013
}

The pinewood nematode (PWN), Bursaphelenchus xylophilus, was introduced in Japan from North America in the early 20th century and rapidly disseminated to China, Taiwan and Korea, most likely via the trade of infected wood (Mota \& Vieira, 2008). It was detected for the first time in Europe (Portugal) in 1999 (Mota et al., 1999) and later in Spain (Abelleira et al., 2011) and the Madeira Island (Fonseca et al., 2012), representing a potential threat to all European conifer forests. In order to characterize the mtDNA lineage(s) present in Europe, three PWN isolates were extracted from Pinus pinaster trees in different regions of Portugal (Santiago do Cacém, Alcácer do Sal and Santa Comba Dão) and maintained as previously described (Fonseca et al., 2008). Total DNA was extracted from each isolate using a standard phenol/chloroform method. A total of 40 PCR primers (available upon request) targeting conserved nematode mtDNA regions were used for amplification, under previously described conditions (Pereira et al., 2013). PCR products were sequenced on an ABI 3130XL Automated Sequencer (Applied Biosystems, Foster City, CA) using a primer-walking strategy. We succeeded

\footnotetext{
*These authors contributed equally to this work.

Correspondence: Filipe Pereira, Institute of Molecular Pathology and Immunology, University of Porto (IPATIMUP), Rua Dr. Roberto Frias s/n, 4200-465 Porto, Portugal. Tel: +351 22 5570700. Fax: +351 22 5570799. E-mail: fpereirapt@gmail.com
}

in sequencing $88 \%(12,945 \mathrm{bp})$ of the mtDNA and identifying the expected 12 protein-coding genes, 22 tRNA genes and two rRNA genes in the three isolates (Supplementary Figure S1; Supplementary Material available at http://www.portugene.com/ SupMat/BxEurope_SM.pdf). All attempts to amplify the 3 ' region of the ND4 gene (164 bp) and the large non-coding region (NCR) were unsuccessful, probably due to the presence of a highly ATrich NCR (AT content of 98.7\%). The three isolates (GenBank JQ429761, JQ514067 and JQ514068) showed no polymorphisms, in agreement with the genetic homogeneity previously observed in Southwestern Europe (Pereira et al., 2013).

We found that the COX3 termination codon (TAA) present in the recently reported mtDNA from a South Korean isolate [GQ332424 (Sultana et al., 2013)] is absent from the Portuguese isolates because of the deletion of a single base (Figure 1A). The same deletion was observed in the partial mtDNA of a Japanese isolate identified in a BLAST search against B. xylophilus contigs available at http://www.genedb.org/ Homepage/Bxylophilus (Kikuchi et al., 2011). Therefore, the COX3 transcripts of Portuguese and Japanese isolates use an incomplete termination codon (ending with ' $\mathrm{TA}$ ') that is presumably converted to TAA by post-transcriptional polyadenylation and/or are extended to the first in-frame stop codon 19 nucleotides downstream, yielding a larger COX3 protein (Figure 1A). The Portuguese/Japanese and Korean isolates also differ considerably in ND4 sequence, where two insertion/deletion (indel) polymorphisms change the predicted protein in a stretch of seven amino acids (Supplementary Figure S2). Moreover, length variation among geographical isolates in a stretch of A nucleotides alters the predicted DHU arm of the tRNA-Tyr (Figures 1B and Supplementary Figure S3). Despite the differences described above, the Portuguese and Korean isolates had the highest 
(A)

Bx Portugal \& Bx Japan

Bx Korea

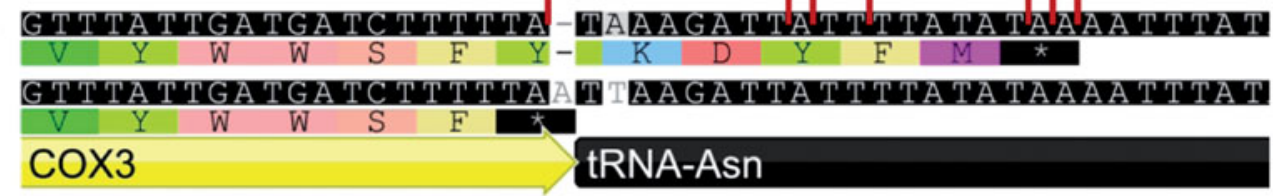

(B)

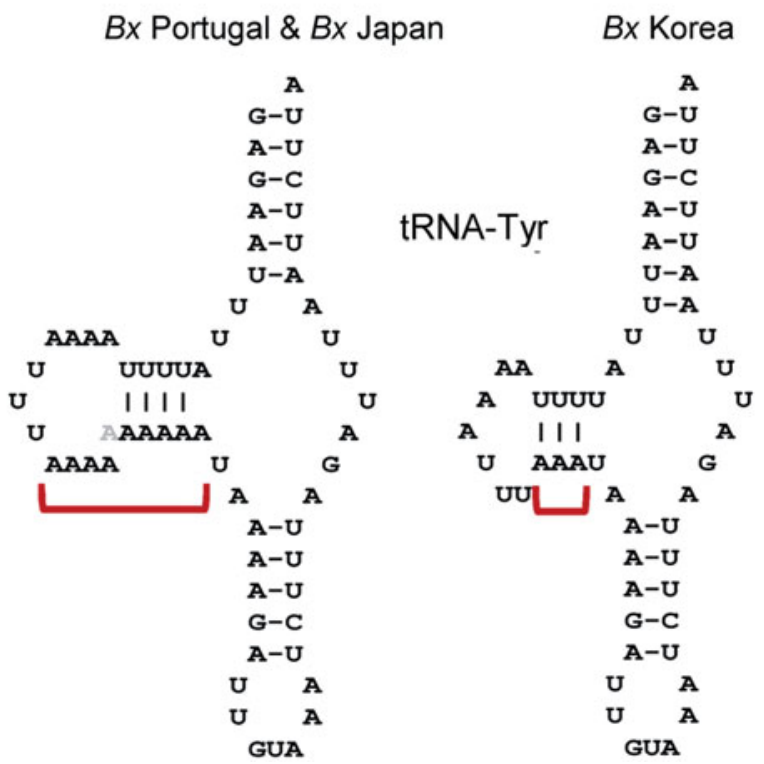

Figure 1. (A) Alignment of the mitochondrial COX3 terminal region in B. xylophilus (Bx). Putative mRNA termination sites are indicated by red bars. The predicted amino acid sequence is shown below the DNA sequences. (B) Predicted secondary structures of the tyrosine tRNA differing due to length variation in the mononucleotide repeat indicated by a red bracket. tRNA secondary structures were drawn using the tRNAscan-SE 1.21 software (http://lowelab.ucsc.edu/tRNAscan-SE/).

percentage of identical sites (99.2\%; Supplementary Figure S4). These contrasting results suggest a complex mutational pattern in the PWN phylogeny.

\section{Acknowledgements}

We are grateful to Paulo Vieira and Pedro Barbosa for helping with the sample collections.

\section{Declaration of interest}

This work was partially supported by the Portuguese "Fundo Florestal Permanente", FEDER (COMPETE) and the Portuguese Foundation for Science and Technology (project PTDC/AGR-CFL/098916/2008 and research grants SFRH/BPD/73108/2010 to BA, SFRH/BD/44264/2008 to IP-C and SFRH/BPD/44637/2008 to FP). MM was partially supported by the EC 7th Framework project REPHRAME (KBBE.2010.1.4-09). The authors report no conflicts of interest. The authors alone are responsible for the content and writing of the paper.

\section{References}

Abelleira A, Picoaga A, Mansilla JP, Aguin O. (2011). Detection of Bursaphelenchus xylophilus, causal agent of pine wilt disease on pinus pinaster in Northwestern Spain. Plant Dis 95:776.
Fonseca L, Cardoso JMS, Lopes A, Pestana M, Abreu F, Nunes N, Mota M, Abrantes I. (2012). The pinewood nematode, Bursaphelenchus xylophilus, in Madeira Island. Helminthologia 49:96-103.

Fonseca L, dos Santos MCV, Santos MS, Curtis RHC, Abrantes IMD. (2008). Morpho-biometrical characterisation of Portuguese Bursaphelenchus xylophilus isolates with mucronate, digitate or round tailed females. Phytopathol Mediterranea 47:223-33.

Kikuchi T, Cotton JA, Dalzell JJ, Hasegawa K, Kanzaki N, McVeigh P, Takanashi T, et al. (2011). Genomic insights into the origin of parasitism in the emerging plant pathogen Bursaphelenchus xylophilus. PLoS Pathogens 7:e1002219.

Mota M, Vieira P. (2008). Pine wilt disease: A worldwide threat to forest ecosystems. Dordrecht: Springer.

Mota M, Braasch H, Bravo MA, Penas AC, Burgermeister W, Metge K, Sousa E. (1999). First report of Bursaphelenchus xylophilus in Portugal and in Europe. Nematology 1:727-34.

Pereira F, Moreira C, Fonseca L, van Asch B, Mota M, Abrantes I, Amorim, A. (2013). New insights into the phylogeny and worldwide dispersion of two closely related nematode species, Bursaphelenchus xylophilus and Bursaphelenchus mucronatus. PLoS One 8:e56288.

Sultana T, Kim J, Lee SH, Han H, Kim S, Min GS, Nadler SA, Park JK. (2013). Comparative analysis of complete mitochondrial genome sequences confirms independent origins of plant-parasitic nematodes. BMC Evol Biol 13:12. 